Résumés des conférences et travaux

\title{
Alain Erlande-Brandenburg (1937-2020)
}

Jean-Michel Leniaud

\section{OpenEdition \\ Journals}

Édition électronique

URL : https://journals.openedition.org/ashp/4048

DOI : $10.4000 /$ ashp.4048

ISSN : 1969-6310

Éditeur

Publications de l'École Pratique des Hautes Études

\section{Édition imprimée}

Date de publication : 1 septembre 2021

Pagination: XVII-XX

ISSN : 0766-0677

\section{Référence électronique}

Jean-Michel Leniaud, "Alain Erlande-Brandenburg (1937-2020) », Annuaire de l'École pratique des

hautes études (EPHE), Section des sciences historiques et philologiques [En ligne], 152 | 2021, mis en ligne le 14 juin 2021, consulté le 23 juin 2022. URL : http://journals.openedition.org/ashp/4048 ; DOI : https://doi.org/10.4000/ashp.4048 


\section{ALAIN ERLANDE-BRANDENBURG}

$(1937-2020)$

$\mathrm{N}$

OTRE collègue Alain Erlande-Brandenburg est décédé le 6 juin dernier, à l'âge de 82 ans. Il était né le 2 août 1937 à Luxeuil-les-Bains où son grand-père maternel exerçait la médecine. Il avait repris le nom de plume de son grandpère paternel, Albert Brandenburg, qui a publié un œuvre romanesque sous le nom d'Albert Erlande. Il comptait aussi parmi ses parents l'égyptologue Eugène Revillout (1843-1913), spécialiste de démotique et, disait-il, Marius Vachon (1860-1928), figure active de l'histoire de Paris et des arts décoratifs au tournant du $\mathrm{XX}^{\mathrm{e}}$ siècle.

Il était entré à la section en 1972 comme chargé de conférences; il en avait obtenu le diplôme deux ans plus tôt sous la direction de Michel Fleury. En 1974, il concluait le parcours classique de l'époque par une élection comme directeur d'études sur l'intitulé : «Archéologie du Moyen Âge », il était à l'époque conservateur au musée de Cluny sous la direction de Francis Salet. Il resta en fonction à la section jusqu'en 2005, soit 31 ans.

Les conditions dans lesquelles il était arrivé dans ce musée méritent d'être racontées car elles dépeignent une tournure d'esprit plutôt rare dans nos milieux de savants. Il était entré à l'École des chartes sur le conseil de Pierre Marot, alors directeur de cette école, avec la ferme intention de devenir conservateur de musée, conviction qu'il s'était forgée au contact d'un grand collectionneur d'art militaire, Jean Brunon (1895-1982). Il suivait parallèlement les cours de l'École du Louvre comme élève agréé, statut qui permettait d'entrer plus facilement dans le corps de conservateur. Il obtint en 1964 le diplôme d'archiviste paléographe et, l'année suivante, celui de l'École du Louvre.

Mais ce parcours, somme toute régulier, s'était soldé par un incident qu'il raconte dans son petit livre de mémoires parus en 2017 sous le titre Parcours d'un conservateur de musée. Brandenburg avait choisi comme sujet de thèse les «funérailles et sépultures royales en France de la fin du VIII ${ }^{\mathrm{e}}$ siècle à 1285 ». Le jury était présidé par l'un de ses professeurs du Louvre qu'il admirait beaucoup, Pierre Pradel, conservateur en chef du département des sculptures. Mais voici que le maitre se prit du plaisir de multiplier les banderilles à l'encontre du disciple. Furieux et vexé, le jeune chartiste lui tourna le dos pendant qu'il parlait et refusa de lui serrer la main à l'issue de la soutenance.

Ce mouvement d'humeur qui anticipait, d'une certaine façon, sur des comportements estudiantins plus fréquents en mai 1968 et années suivantes fut reçu avec la froideur qui convenait : l'accès au département des sculptures lui fut fermé. Par chance, la providence des chartistes en rupture de ban étendit son bras : Michel Fleury, alors secrétaire de la section, avait pris pendant la soutenance le parti du rebelle. Et lui trouva un séjour dans un purgatoire qui n'avait rien d'inconfortable : les fossés du Louvre. Comme chercheur au CNRS, Brandenburg fut attaché à l'équipe de recherche qui fouillait au pied de la colonnade et devant la Seine dans le but d'y creuser les fossés dont Malraux avait ordonné la réalisation. Il y trouva les substructions d'un 
projet inabouti qu'il put attribuer à Louis Le Vau. L'expression «Archéologie du Moyen Âge » qui a désigné sa conférence à la section ne renvoyait pas seulement à la chaire fondatrice de Jules Quicherat à l'École des chartes, « archéologie médiévale », mais à cette première expérience professionnelle.

J'ai fréquenté la conférence d'Alain Brandenburg entre 1974 et 1976. Il n'était pas seulement connu, à l'époque, comme conservateur au musée de Cluny, mais comme pilier de la Société française d'archéologie, dont le dynamisme devait aussi beaucoup au charisme de son président, Jean Chatelain, alors directeur des musées de France - il en fut le successeur de 1985 à 1994. Il y assurait la dure fonction de directeur du Bulletin monumental et y fonda une collection destinée à la publication des thèses les plus remarquées : la Bibliothèque de la Société française d'archéologie. Son charisme attirait une trentaine de personnes qui s'entassaient dans la petite salle Delamare, aujourd'hui détruite au profit d'un immonde escalier de secours : beaucoup venaient du séminaire de Louis Grodecki à Paris IV, quelques-uns de l'École des chartes ou de l'École du Louvre. En ce lieu surchauffé, on ne fumait pas, heureusement; on se serait néanmoins cru dans un séminaire de la sixième section. Le directeur d'études y transpirait lui-même si généreusement que sa veste de velours bleu marine, qu'il portait par tous les temps quand il était encore célibataire, en était décolorée. C'est en ce lieu que son œuvre s'est construit, un œuvre considérable, qui abordait aussi bien des points d'érudition : datation, attribution, localisation de sculptures médiévales, selon les méthodes éprouvées du catalogage muséographique, que des réflexions de synthèse. L'étude de l'Adam de Notre-Dame, aujourd'hui au musée de Cluny, du jubé de la même cathédrale, d'un gisant de Saint-Germain-des-Près, précédait celle de la façade de la cathédrale d'Amiens. Plus tard, Alain Brandenburg entreprit de construire une réflexion sur un gigantesque sujet : la cathédrale depuis les temps paléo-chrétiens.

Il écrivit au total plus de 400 articles et pas loin d'un vingtaine de livres, dont Le Roi est mort. Études sur les funérailles, les sépultures des rois de France jusqu'à la fin du XIII siècle (1975), La Dame à La Licorne (1978), L'Art gothique (1983), Le Monde gothique. La conquête de l'Europe. 1260-1300 (1987), La Cathédrale (1989), Notre-Dame de Paris (1991), De pierre, d'or et de feu. La création artistique au Moyen Âge (1999).

Brandenburg n'aimait pas trop le monde universitaire mais il adorait l'enseignement. Pas seulement à la section, mais à l'École du Louvre où il fit fonder par Dominique Ponnau, le directeur de l'époque, la chaire d'histoire de l'architecture, à l'École supérieure de Chaillot et à l'École des chartes. En ce dernier lieu, au départ de Jacques Thirion en 1992, il obtint d'Emmanuel Poulle que l'antique et fameuse chaire de Jules Quicherat d'archéologie médiévale fût transformée en trois chaires d'histoire de l'art : Moyen Âge, Temps modernes et Époque contemporaine. De cette réforme, confirmée ensuite par Yves-Marie Bercé, hélas fortement contestée en interne ces derniers temps, il est résulté une transformation radicale du profil et du recrutement des conservateurs aspirant à servir dans les musées et les monuments historiques.

Parallèlement à ces activités savantes, Brandenburg poursuivit une féconde carrière administrative et muséographique. De 1987 à 1991, il exerça la fonction d'adjoint au directeur des musées de France. Il était coutumier à l'époque aux Musées 
que le directeur, généralement recruté dans les corps sortis de l'ENA, fût épaulé par un membre de l'inspection générale : Alain Brandenburg fut l'adjoint d'Olivier Chevrillon puis de Jacques Sallois. De cette époque, je retiendrai l'appui qu'il apporta à la création du corps unique des conservateurs du patrimoine selon le dessein que Jean-Ludovic Silicani avait conçu en accompagnement de ce qui allait devenir l'Institut national du patrimoine.

En 1994, il fut choisi pour succéder à notre ancien collègue, Jean Favier, à la tête de la direction des archives. La mission était difficile : vingt ans de direction, longévité exceptionnelle dans la haute administration, n'avait pas contribué à faire du palais Soubise un haut lieu de la modernisation de l'État. Les quatre années que le nouveau directeur consacra à la fonction furent difficiles. Il parvint à réorganiser son administration centrale et à déconcentrer les compétences; il projeta, dans la suite du rapport commandé au conseiller d'État Guy Braibant, la création d'un centre des archives de la Cinquième République qui aurait été installé à Reims. Les protestations qui accompagnèrent la nouveauté de la proposition et le retard que Catherine Trautmann, nommée ministre de la Culture en 1997, mit à approuver ses projets le conduisirent en 1998 à démissionner d'un poste que son enthousiasme n'avait pas suffi à rendre moins ingrat. La cité interministérielle des archives à Pierreffitte-sur-Seine reste néanmoins l'héritière partielle de ses ambitions.

En 1998, il reprit la fonction qu'il aimait plus que tout, celle de conservateur. Il choisit de consacrer ses dernières années au musée d’Écouen. Il en avait été le père.

De cette histoire tout commence en fait à Cluny : il en avait enrichi les collections en suscitant plusieurs acquisitions, par exemple celle d'une tête de prophète provenant de l'abbatiale de Saint-Denis, qu'après vingt ans de tractations il fit acheter à l'un de ses auditeurs à la section. Il y mit en valeur la collection d'art juif dont l'essentiel venait de l'ensemble réuni par le musicien Isaac Strauss (arrière-grand-père de Claude Lévy-Strauss) et racheté par la baronne Nathaniel de Rothschild pour être légué au musée, la fit exposer au Grand Palais en 1981 et à Jérusalem puis, avec Victor Klagsbald et la fille de ce dernier, Laurence Sigal, entreprit les premières démarches pour la création du musée d'Art juif dans l'hôtel de Saint-Aignan.

Vers la même époque, en 1977, François Giscard d'Estaing, alors président de la banque française du commerce extérieur, le contactait : ce fut le point de départ d'une extraordinaire aventure. Ce dernier venait de découvrir dans les fondations de l'hôtel particulier où se trouvait la direction de la banque, un rassemblement considérable de têtes de rois gothiques. Ses recherches le convainquirent qu'elles avaient été entassées là en 1796 par Jean-Baptiste Lakanal, frère de Joseph le conventionnel, et qu'elles provenaient de la décapitation des rois de la galerie occidentale en 1793. Il s'était aussi persuadé qu'elles devaient être installées à Cluny. Il s'y rendit dans l'idée d'avancer dans son projet. Immédiatement, des obstacles lui furent opposés : le directeur du musée, Francis Salet, mit en avant le manque de place; Michel Fleury tenta de faire attribuer les têtes à Carnavalet. Par chance, Brandenburg, jeune et enthousiaste, brava la disgrâce de ses deux maîtres et se chargea de mettre en œuvre le dessein de François Giscard d'Estaing. Il en résulta la salle Notre-Dame de Paris en 1982.

Dans le même temps, il conduisait un projet encore plus ambitieux. La Grande Chancellerie de la Légion d'honneur ayant abandonné le bâtiment d'Écouen qui recevait jusqu'alors le pensionnat de jeunes filles, il avait été décidé sur la proposition de 
Pierre Verlet d'y installer un musée de la Renaissance française en y transférant les collections correspondantes du musée de Cluny. Malgré le soutien d'André Malraux, le projet n'avançait pas et, au début des années 1970, le président Pompidou, épris de culture, voulut confier le bâtiment au groupe Trigano pour y installer un club de loisirs. Il s'en suivit une bataille d'opinion d'où le projet de musée de la Renaissance française sortit vainqueur. Brandenburg s'introduisit dans le processus de décision et de conduite des travaux. Le 25 octobre 1977, peu de temps après l'affaire des têtes de Notre-Dame, le Président Valéry Giscard d'Estaing venait inaugurer le nouveau musée. Cinq jours plus tôt, le 25 octobre, le même avait annoncé qu'il créait le musée d'Orsay.

À ce musée d'Écouen dont il avait conçu la muséographie, Alain Brandenburg resta charnellement attaché et le choisit pour théâtre de ses dernières fonctions.

Notre collègue ne fut pas seulement un professeur, un savant, un conservateur, un homme d'action. Il reçut des honneurs, qu'il ne briguait pas et qu'il ne détestait pas, et des charges lui furent confiées. Franck Riester, ministre de la Culture, qui le connaissait pour l'avoir consulté sur des question patrimoniales à Coulommiers, lui a rendu un bel hommage. C'était surtout un tempérament : prompt à l'enthousiasme mais aussi à la colère, généreux, méprisant l'esprit d'intrigue et l'ambition de ceux qui s'emparent du pouvoir dans le seul projet de le conserver, il avait l'amitié féconde. Son œuvre passera sans doute, de même que les nôtres, mais il restera comme le souffle d'un grand dessein. 